\title{
Study of growth, metabolism, and morphology of Akkermansia muciniphila with an in vitro advanced bionic intestinal reactor
}

\author{
Zhitao Lii, Guoao Hu' ${ }^{1}$ Li Zhu'², Zhenglong Sun ${ }^{3}$, Yun Jiang ${ }^{1}$, Min-jie Gao ${ }^{1 *}$ and Xiaobei Zhan ${ }^{1 *}$ (1)
}

\begin{abstract}
Background: As a kind of potential probiotic, Akkermansia muciniphila abundance in human body is directly causally related to obesity, diabetes, inflammation and abnormal metabolism. In this study, A. muciniphila dynamic cultures using five different media were implemented in an in vitro bionic intestinal reactor for the first time instead of the traditional static culture using brain heart infusion broth (BHI) or BHI + porcine mucin (BPM).

Results: The biomass under dynamic culture using BPM reached $1.92 \mathrm{~g} / \mathrm{L}$, which improved $44.36 \%$ compared with the value under static culture using BPM. The biomass under dynamic culture using human mucin (HM) further increased to the highest level of $2.89 \mathrm{~g} / \mathrm{L}$. Under dynamic culture using porcine mucin (PM) and HM, the main metabolites were short-chain fatty acids (acetic acid and butyric acid), while using other media, a considerable amount of branchedchain fatty acids (isobutyric and isovaleric acids) were produced. Under dynamic culture Using HM, the cell diameters reached $999 \mathrm{~nm}$, and the outer membrane protein concentration reached the highest level of $26.26 \mu \mathrm{g} / \mathrm{mg}$.
\end{abstract}

Conclusions: This study provided a preliminary theoretical basis for the development of A. muciniphila as the next generation probiotic.

Keywords: Akkermansia muciniphila, Anaerobic fermentation, Bioreactor, Short-chain fatty acids

\section{Background}

In recent years, the research and the development of Akkermansia muciniphila has attracted interest [1-5]. A. muciniphila is an elliptical, immobile, strictly anaerobic Gram-negative strain that can use mucin in the intestine as carbon, nitrogen, and energy sources for growth [6]. The main metabolite of $A$. muciniphila are short-chain fatty acids (SCFAs) and branched-chain fatty acids (BCFAs) [7-10]. A. muciniphila was considered as a potential probiotic because evidences proved that $A$.

\footnotetext{
* Correspondence: gaominjie@msn.com; xbzhan@yahoo.com

${ }^{1}$ Key Laboratory of Carbohydrate Chemistry and Biotechnology, Ministry of Education, School of Biotechnology, Jiangnan University, Wuxi 214122, China Full list of author information is available at the end of the article
}

muciniphila had a causal relationship with obesity [11-14], diabetes [15-17], inflammation [18, 19], autism $[20,21]$, amyotrophic lateral sclerosis [22], premature aging [23], epilepsy [24], hypertension [25], cancer [26] and metabolic abnormalities [27]. Recent studies reported that butyric acid produced by intestinal microbes like $A$. muciniphila could promote $\beta$ cells to secrete insulin and then regulate blood sugar [28]. In addition, A. muciniphila cells with thicker outer membrane protein (Amuc-1100) showed better effects in preventing obesity and related complications [14].

In terms of in vitro culture of $A$. muciniphila, the traditional way was to use brain heart infusion broth (BHI) or $\mathrm{BHI}+$ porcine mucin $(\mathrm{BPM})$ in an anaerobic bottle

C C The Author(s). 2021 Open Access This article is licensed under a Creative Commons Attribution 4.0 International License, which permits use, sharing, adaptation, distribution and reproduction in any medium or format, as long as you give appropriate credit to the original author(s) and the source, provide a link to the Creative Commons licence, and indicate if changes were made. The images or other third party material in this article are included in the article's Creative Commons licence, unless indicated otherwise in a credit line to the material. If material is not included in the article's Creative Commons licence and your intended use is not permitted by statutory regulation or exceeds the permitted use, you will need to obtain permission directly from the copyright holder. To view a copy of this licence, visit http://creativecommons.org/licenses/by/4.0/ The Creative Commons Public Domain Dedication waiver (http://creativecommons.org/publicdomain/zero/1.0/) applies to the data made available in this article, unless otherwise stated in a credit line to the data. 
and placed it in an anaerobic environment for static fermentation culture [29-31]. Porcine mucin (PM) was the key component of $A$. muciniphila media in most reports. By adding a certain amount of mucin to BHI to cultivate A. muciniphila, the nutritional requirements for its growth and metabolism could be met. For simulation of A. muciniphila physiological state in human body, using human mucin (HM) instead of PM for in vitro culture is one key issue of great significance. However, as far as we know, the in vitro culture of $A$. muciniphila using HM has not been reported yet.

For culture method, the currently used A. muciniphila static culture is easy to cause accumulation of metabolites, which in turn inhibits the growth of A. muciniphila. In addition, A. muciniphila cells are difficult to harvest because many of them adhere to bottle bottom and wall. The in vitro dynamic fermentation reactor is a recently developed tool for studying the intestinal flora, mainly through bionic technology to simulate the real environment in digestive system [32]. In this study, an advanced bionic intestinal reactor (ABIR) was built to simulate the peristalsis of the real human colon, the micro-ecological environment of intestinal and the absorption of flora metabolites. As the traditional static culture state is far from the real $A$. muciniphila growth environment in human body, using ABIR as the $A$. muciniphila culture equipment is another key issue of great significance for simulating in vivo A. muciniphila physiological environment.
In this study, in order to better understand the growth, metabolism and morphology of A. muciniphila in real human body, in vitro dynamic $A$. muciniphila fermentation was implemented using five different media including $\mathrm{HM}$ and $\mathrm{BHI}+$ human mucin (BHM) in ABIR. The discovery was an improvement in culture method and media for the development of $A$. muciniphila as the next generation probiotic.

\section{Results}

Effects on cell growth and metabolites of A. muciniphila under static and dynamic cultures

As shown in Fig.1a, the logarithmic growth periods of static cultures and ABIR dynamic culture were both $6-10 \mathrm{~h}$, then the bacteria entered a stable stage after $12 \mathrm{~h}$. The biomass under the ABIR dynamic culture increased steadily after $37 \mathrm{~h}$ and reached $1.92 \mathrm{~g} / \mathrm{L}$ at $48 \mathrm{~h}$, which was $44.36 \%$ higher than that of the static culture $(1.33 \mathrm{~g} / \mathrm{L})$. For both static and dynamic cultures, the type and concentration of metabolites were shown in Fig. 1b. Under dynamic culture, the metabolites were mainly acetic acid, propionic acid, butyric acid, isobutyric acid, and isovaleric acid yields, which reached $47.05,10.21,9.87,2.78$, and $4.06 \mathrm{mM}$, respectively. The differences in yields of butyric acid between two cultures were significant (one-way ANOVA analysis with Tukey's test, $p<0.05$, the same below). Simultaneously, considerable differences were

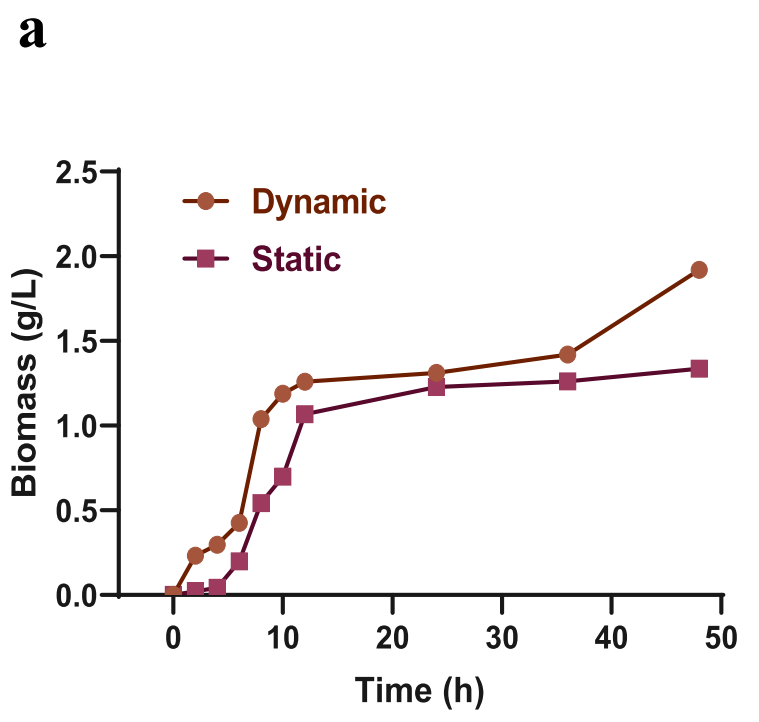

b

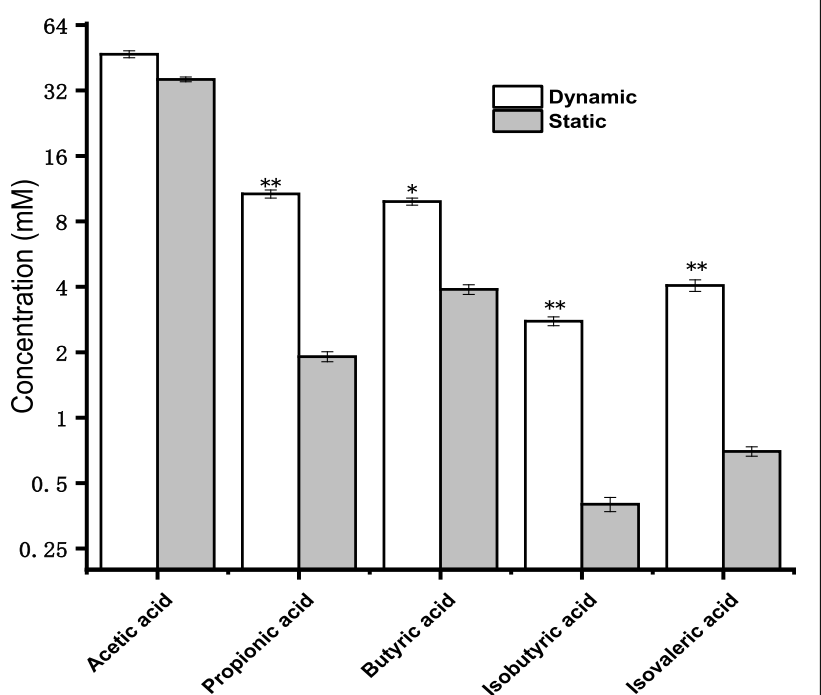

Fig. 1 Effects on cell growth and metabolites of A. muciniphila under static culture and dynamic culture. Note: The data are shown as the mean $\pm \mathrm{SD}(n=3)$ and analyzed using one-way ANOVA with Tukey's test, ${ }^{*} p<0.05,{ }^{* *} p<0.01$ 

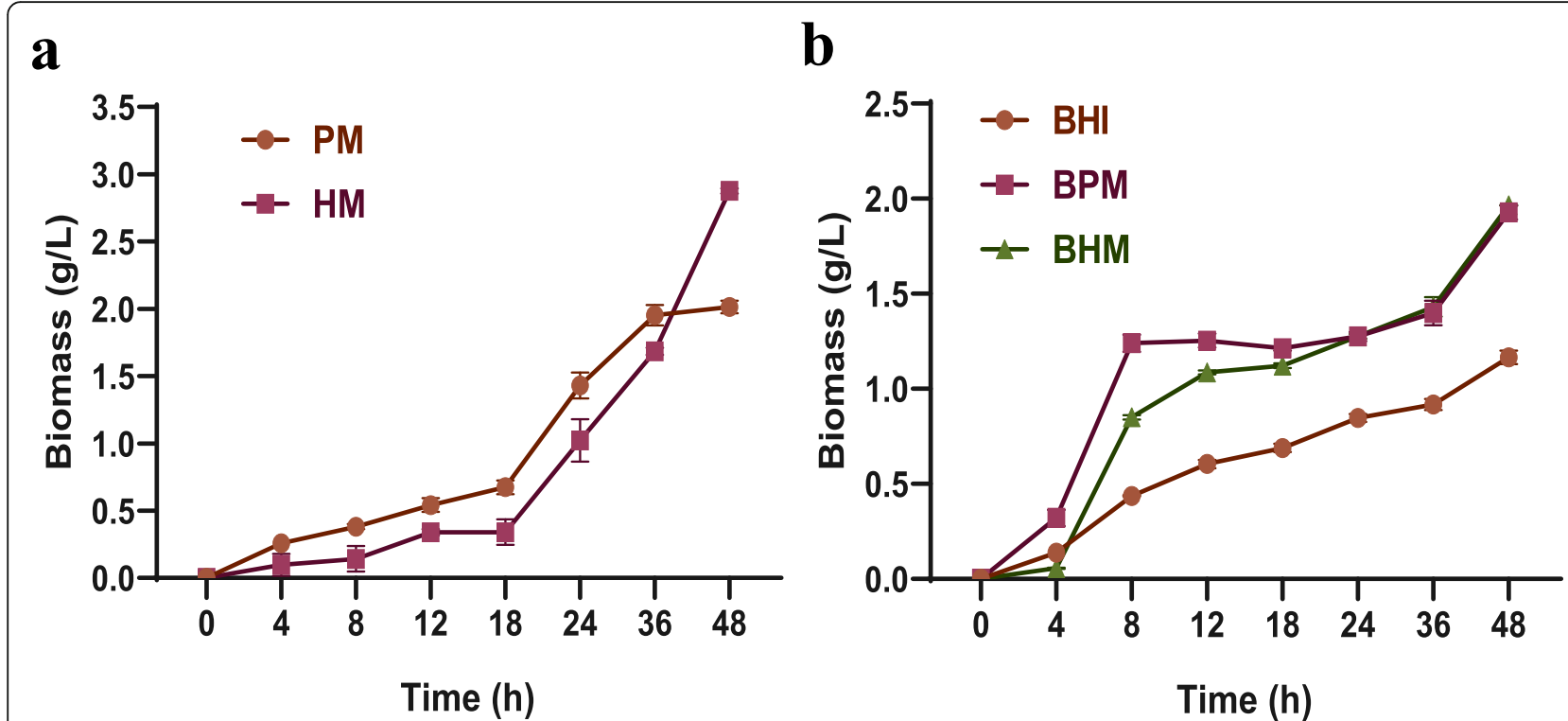

Fig. 2 Effects of different media on A. muciniphila growth under dynamic culture. In this figure, the brain heart infusion broth (BHI), porcine mucin (PM), human mucin (HM), BHI+ porcine mucin (BPM), and BHI+ human mucin (BHM)

observed in terms of yields of propionic acid, isobutyric acid and isovaleric acid $(p<0.01)$.

Effects of different media on A. muciniphila growth under dynamic culture

As shown in Fig. 2a, using PM and HM, A. muciniphila grew slowly at $0-18 \mathrm{~h}$, and the biomass of $A$. muciniphila grown on PM $(0.68 \mathrm{~g} / \mathrm{L})$ was higher than that on HM $(0.35 \mathrm{~g} / \mathrm{L})$. During this period, the secretion rates of glycosidase and protease accelerated, resulting in increased degradation of mucin. After this period, $A$. muciniphila adapted to the growth environment of medium. The growth rates of $A$. muciniphila on PM and HM were both in the logarithmic period from $18 \mathrm{~h}$ to $36 \mathrm{~h}$. From 36 to $48 \mathrm{~h}$, the biomass of $A$. muciniphila on HM exceeded that on PM and reached $2.89 \mathrm{~g} / \mathrm{L}$ at $48 \mathrm{~h}$. The results proved that $A$. muciniphila culture medium based on human mucin was an ideal medium for cell growth. Moreover, growth performances of $A$. muciniphila on BHI, BPM, and BHM were studied. As shown in Fig. $2 \mathrm{~b}$, the $A$. muciniphila grown on BPM and BHM were in logarithmic growth phase at $4-8 \mathrm{~h}$, and the $A$. muciniphila biomass on BPM $(1.26 \mathrm{~g} / \mathrm{L})$ was higher than that on BHM $(0.85 \mathrm{~g} / \mathrm{L})$. The A. muciniphila grown on BHI was in the logarithmic growth phase at 8-12 $\mathrm{h}$, and $A$. muciniphila biomass of $0.61 \mathrm{~g} / \mathrm{L}$ was obtained, which was lower than that on BPM and BHM. All three batches subsequently entered a stable growth phase. At $48 \mathrm{~h}$, the biomass on BHI, BPM, and $\mathrm{BHM}$ were 1.17, 1.92, and $1.96 \mathrm{~g} / \mathrm{L}$, respectively.
Effects of different media on A. muciniphila metabolites under dynamic culture

As shown in Fig. 3. The metabolites of A. muciniphila on PM and HM were SCFAs (acetic acid and butyric acid), whereas the metabolites of $A$. muciniphila on $\mathrm{BHI}$, BPM, and BHM were SCFAs (acetic, propionic, butyric, valeric) and BCFAs (isobutyric and isovaleric acids). For the batches grown on PM and HM, the concentrations of acetic acid (4-6 mM) were not significantly different (Fig. 3b), but the concentrations of butyric acid were significantly different (Fig. 3a, $p<0.05$ ). The concentrations of butyric acid reached $1.06 \mathrm{mM}$ (batch on $\mathrm{HM}$ ) and $0.68 \mathrm{mM}$ (batch on PM). For the batches grown on BHI and BHM, the concentrations of acetic acid $(50.43 \mathrm{mM}$ and $22.81 \mathrm{mM})$ were significantly different $(p<0.05)$ (Fig. 3c). The concentrations of isobutyric acid (3.15 mM and $2.88 \mathrm{mM}$ ) were significantly different (Fig. 3e, $p<$ $0.05)$. The concentrations of isovaleric acid $(4.25 \mathrm{mM}$ and $4.07 \mathrm{mM}$ ) were significantly different (Fig. $3 \mathrm{~g}, p<$ 0.05). The concentrations of valeric acid were very significantly different (Fig. 3h, $p<0.001$ ). For the batches grown on BHI and BPM, the concentrations of valeric acid were very significantly different $(p<0.01)$. For the batches grown on BPM and BHM, the concentrations of valeric acid were very significantly different $(p<0.05)$. For the batches grown on BHI, BPM and BHM, the concentrations of propionic acid were no significant difference (Fig. 3d, $p>0.05$ ). The concentrations of butyric acid $(5.79 \mathrm{mM}, 9.88 \mathrm{mM}$ and $12.88 \mathrm{mM})$ were significantly different (Fig. 3f, $p<0.05$ ). The concentrations of valeric acid $(0.14 \mathrm{mM}, 0.41 \mathrm{mM}$, and $0.51 \mathrm{mM})$ were significantly different (Fig. 3h, $p<0.01$ ). 


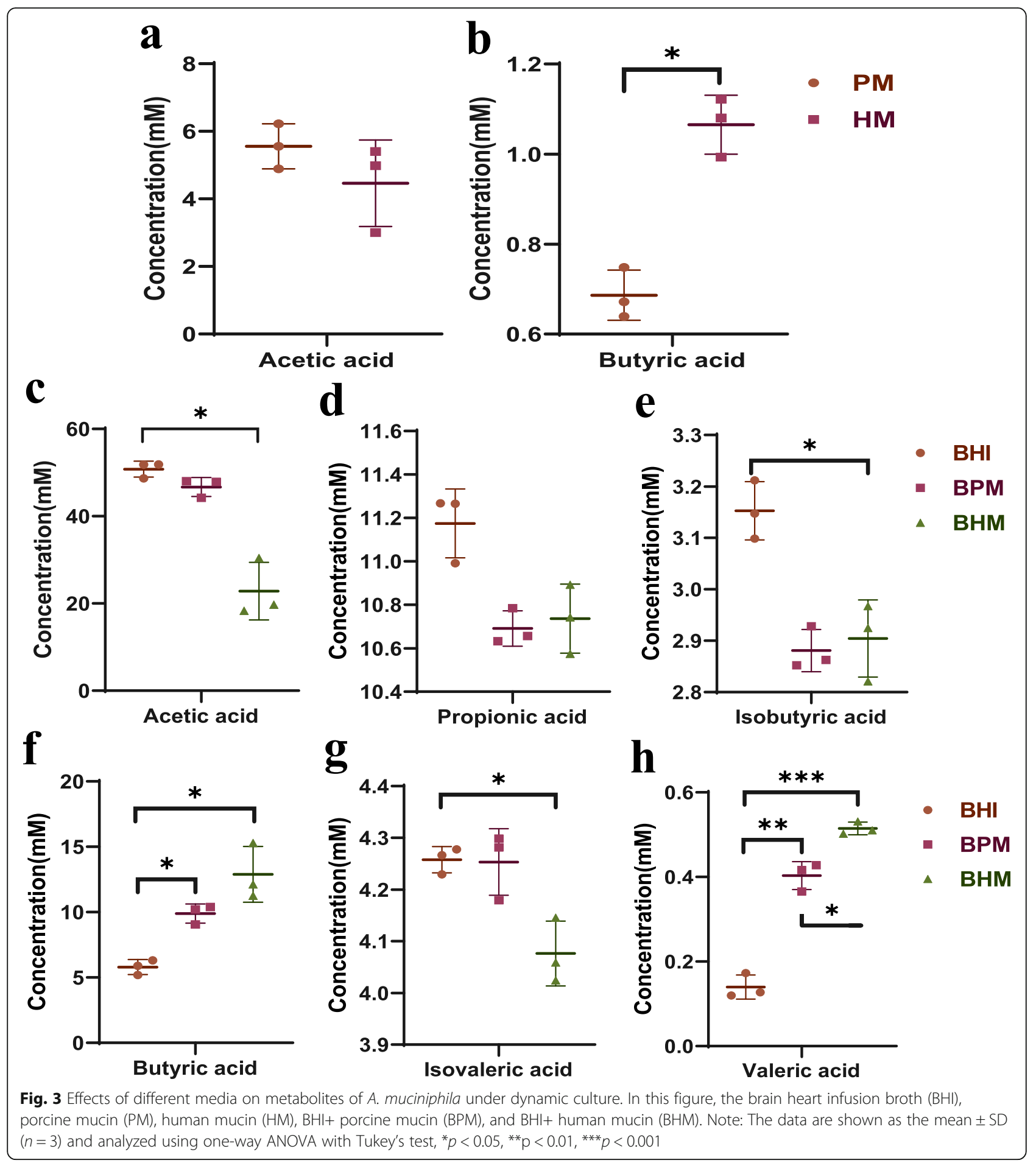

Effects of different media on the concentration of outer membrane protein under dynamic culture

As shown in Fig. 4, the outer membrane protein concentrations of A. muciniphila in the batches grown on BHI, PM, HM, BPM, and BHM were 17.03, 24.36, 26.26, 18.34, and $19.45 \mu \mathrm{g} / \mathrm{mg}$, respectively. Significant differences between the BHI, BPM, BHM, PM and HM were observed $(p<0.05)$.
Effects of different media on the thickness of $A$. muciniphila outer membrane and diameter of cell under dynamic culture

Figure 5 showed the TEM and SEM images of $A$. muciniphila using five different culture media under dynamic culture. The cells of $A$. muciniphila were round or elliptical. The cells of the batch grown on BHI grew alone or 


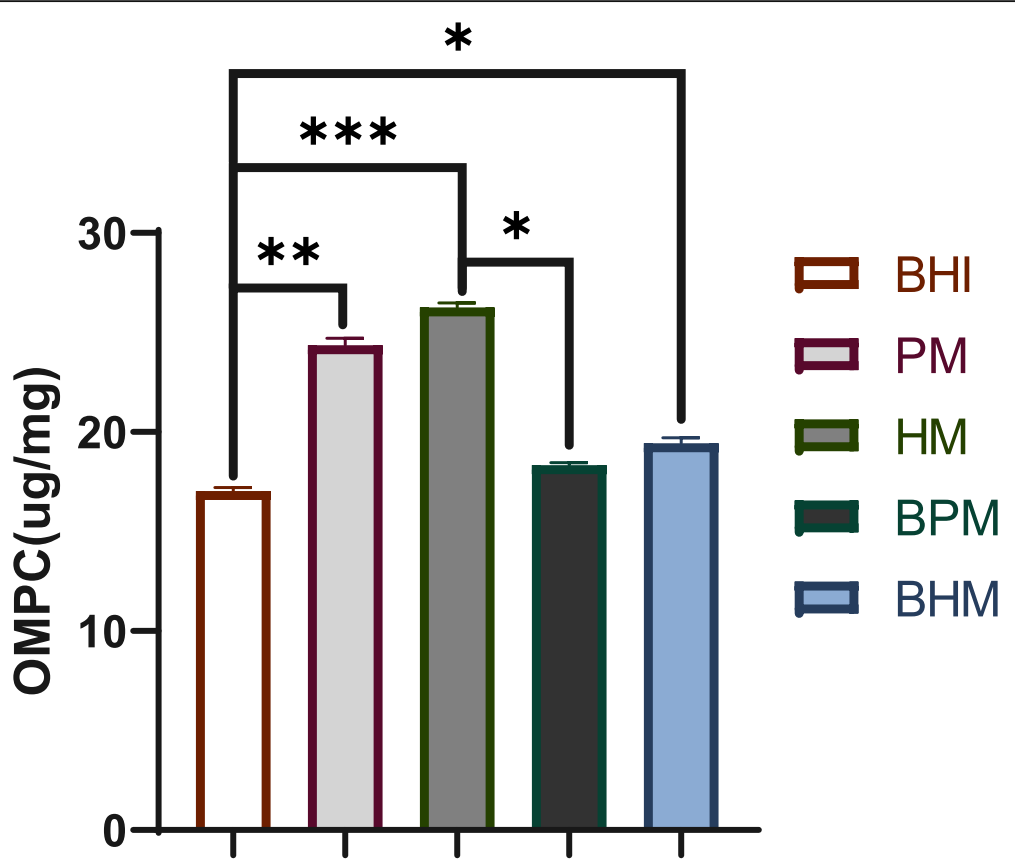

Fig. 4 Effects of different media on concentration of A. muciniphila outer membrane protein under dynamic culture. In this figure, the brain heart infusion broth $(\mathrm{BHI})$, porcine mucin (PM), human mucin (HM), BHI+ porcine mucin (BPM), and BHI+ human mucin (BHM). Note: The data are shown as the mean \pm SD $(n=3)$ and analyzed using one-way ANOVA with Tukey's test, ${ }^{*} p<0.05,{ }^{* *} p<0.01,{ }^{* * *} p<0.001$

in pairs, whereas the cells of the batches grown on PM, $\mathrm{HM}, \mathrm{BPM}$, and BHM containing mucin grew in pairs or chains and even formed aggregates. As shown in Table 1, the relative thicknesses of the outer membranes of $A$. muciniphila in the BHI, PM, HM, BPM, and BHM were 71.66, 101.20, 104.50, 71.46, and $72.11 \mathrm{~nm}$, respectively. Significant differences between the BHI and PM, PM and BPM, PM and BHM, BHI and HM, HM and BPM, and $\mathrm{HM}$ and $\mathrm{BHM}$ were observed $(p<0.01)$. No significant difference was observed in the other remaining groups $(p>0.05)$. The above results were consistent with those of the outer membrane protein concentration of A. muciniphila, indicating that increased outer membrane thickness of the cells resulted in high outer membrane protein concentration. The cell diameters of $A$. muciniphila grown on BHI, PM, HM, BPM, and BHM were $871,985,999,980$, and $971 \mathrm{~nm}$, respectively. The cell diameter on BHI was significantly different with those on the other four media $(p<0.05)$, and no significant difference was observed between the other groups $(p>0.05)$. On the mucin medium, A. muciniphila had a diameter and length of 640 and $690 \mathrm{~nm}$, respectively. On BHI medium, A. muciniphila had a diameter and length of $830 \mathrm{~nm}$ and $1000 \mathrm{~nm}$, respectively.

\section{Discussion}

The static culture and ABIR dynamic culture were compared in terms of biomass and metabolites. Compared with the static culture, the ABIR simulated physic- chemical and physiological processes in the real human colon, which was an idea tool for investigating the effects of the media components on A. muciniphila fermentation. In static culture, the metabolites of $A$. muciniphila accumulated partially at the late stage of the culture process and high level of SCFAs $(>80 \mathrm{mM})$ inhibited cell growth $[8,33]$. In ABIR dynamic culture, simulation of real human colon peristalsis was achieved, and the cells, media and metabolites could be well mixed. Thus, the inhibition effects of metabolites could largely be released. Under dynamic culture, isobutyric acid and isovaleric acid yields were relatively higher than those under static culture. In the late fermentation stage of dynamic culture, the carbon source was exhausted and A. muciniphila turned to protein fermentation, thereby generated corroded toxic metabolites, such as isobutyric acid and isovaleric acid [34, 35].

Using five different media (BHI, PM, HM, BPM and BHM), A. muciniphila biomass under dynamic cultures were compared. The mucin in the PM and HM were the only carbon and nitrogen sources for the growth of $A$. muciniphila. When A. muciniphila grew on these media, glycosidase was secreted to degrade the glycoprotein exposed at the terminal of mucin to produce $\mathrm{N}$ acetylgalactosamine, $\mathrm{N}$-acetylglucosamine, fucose, and galactose components, which could be used as nutrients for cells [36-38]. The BHI contained rich carbon and nitrogen sources, which supposed to fully support the growth of A. muciniphila. However, the biomass on BHI 


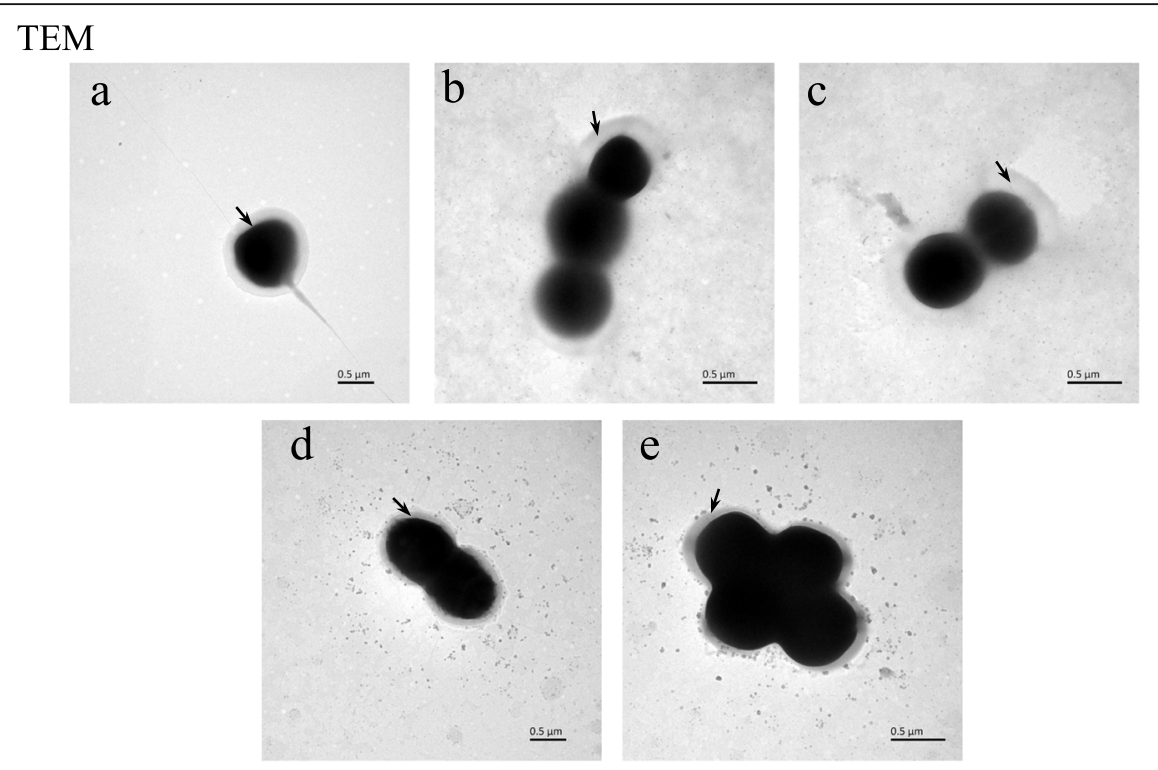

SEM
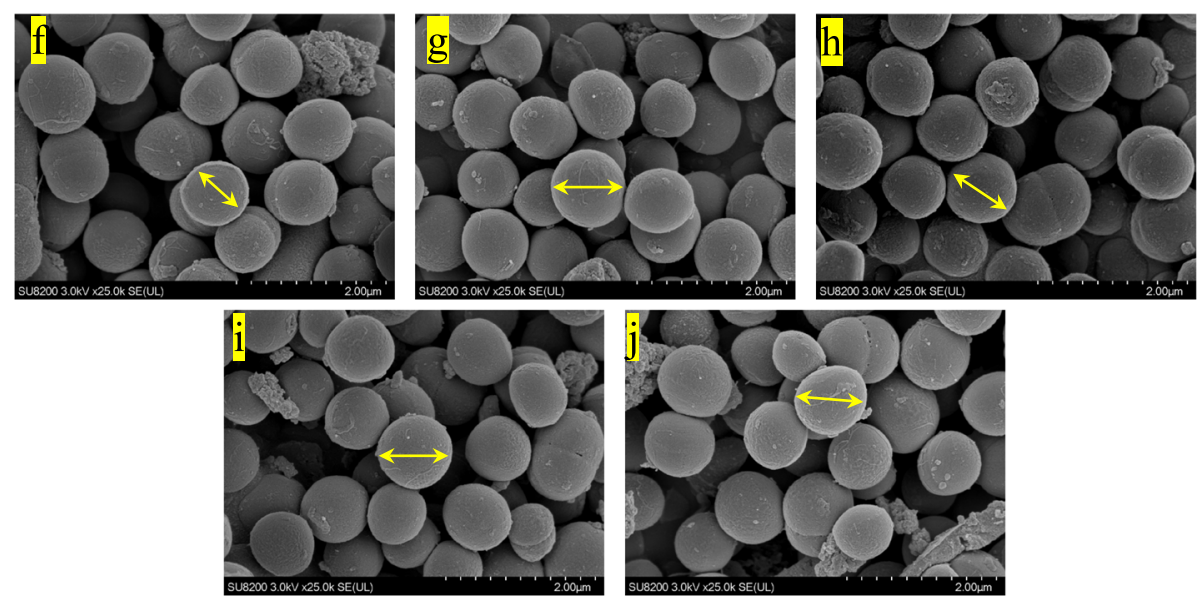

Fig. 5 SEM and TEM images of A. muciniphila on different media under dynamic culture. In this figure, $\mathbf{a}, \mathbf{b}, \mathbf{c}$, $\mathbf{d}$, e represents SEM images of the brain heart infusion broth $(B H I)$, porcine mucin (PM), human mucin (HM), BHI+ porcine mucin (BPM), and BHI+ human mucin (BHM), respectively. $\mathbf{f}, \mathbf{g}, \mathbf{h}, \mathbf{i}, \mathbf{j}$ represents TEM images of the brain heart infusion broth (BHI), porcine mucin (PM), human mucin (HM), BHI+ porcine mucin (BPM), and $\mathrm{BHI}+$ human mucin $(\mathrm{BHM})$, respectively

was the lowest among the three batches. When mucin was added in BHI, the biomass of A. muciniphila increased about $65 \%$, which demonstrated that mucin was a key component for A. muciniphila growth. To find other cheaper sources instead of mucin, Derrien and Colleagues discovered that A. muciniphila could also grow on a limited amount of carbon source, including $\mathrm{N}$-acetylglucosamine, $\mathrm{N}$-acetylgalactosamine, and glucose $[6,31]$. Based on this, a mixed medium instead of mucin to cultivate $A$. muciniphila was formed by adding glucose, soy protein, threonine, $\mathrm{N}$-acetylglucosamine and $\mathrm{N}$-acetylgalactosamine together. The biomass of $A$. muciniphila grown on the mixed medium reached the same level of the batches grown on BPM or BHM [14, 39]. In addition, studies found that adding fructooligosaccharides [40, 41], metformin [42, 43], polyphenols [44-47], probiotics [48-50] and fish oil unsaturated fatty acids [51] could increase the abundance of in vivo A. muciniphila.

Using five different media, the type and the concentration of A. muciniphila metabolites (SCFAs and BCFAs) were further studied, and an interesting conclusion was obtained. At HM and PM, the metabolites of A. muciniphila are acetic and butyric acids. The latest research showed the causal relationship between the butyric acid produced by intestinal microorganisms and the risk of diabetes [28]. Butyric acid can promote the secretion of insulin by $\beta$ cells, regulate blood sugar, and improve insulin response [34, 35]. Further research was needed on the mechanism of $A$. muciniphila main metabolites 
Table 1 Comparative analysis of relative thickness and diameter of A. muciniphila outer membrane on different media under dynamic culture

\begin{tabular}{|c|c|c|c|c|}
\hline Tukey's multiple comparisons test & Mean 1 & Mean 2 & Summary & Adjusted $P$ Value \\
\hline a: BHI vs. PM & 70.66 & 101.2 & * & 0.0159 \\
\hline a: BHI vs. HM & 70.66 & 104.5 & $* *$ & 0.0072 \\
\hline a: BHI vs. BPM & 70.66 & 71.46 & ns & $>0.9999$ \\
\hline a: BHI vs. BHM & 70.66 & 72.11 & ns & $>0.9999$ \\
\hline a: PM vs. HM & 101.2 & 104.5 & ns & 0.9934 \\
\hline a: PM vs. BPM & 101.2 & 71.46 & * & 0.0177 \\
\hline a: PM vs. BHM & 101.2 & 72.11 & * & 0.0152 \\
\hline a: HM vs. BPM & 104.5 & 71.46 & $* *$ & 0.008 \\
\hline a: HM vs. BHM & 104.5 & 72.11 & $* *$ & 0.0068 \\
\hline a: BPM vs. BHM & 72.11 & 72.11 & ns & $>0.9999$ \\
\hline b: BHI vs. PM & 871 & 985 & * & 0.0137 \\
\hline b: BHI vs. PM & 871 & 999.3 & $* *$ & 0.0035 \\
\hline b: BHI vs. BPM & 871 & 980 & $*$ & 0.0136 \\
\hline b: BHI vs. BHM & 871 & 971.7 & * & 0.0214 \\
\hline b:PM vs. HM & 985 & 999.3 & ns & 0.4533 \\
\hline b:PM vs. BPM & 985 & 980 & ns & 0.9623 \\
\hline b:PM vs. BHM & 985 & 971.7 & ns & 0.589 \\
\hline b:HM vs. BPM & 999.3 & 980 & ns & 0.5442 \\
\hline b:HM vs. BHM & 999.3 & 971.7 & ns & 0.3824 \\
\hline b: BPM vs. BHM & 980 & 971.7 & ns & 0.1124 \\
\hline
\end{tabular}

Note: a: Relative thickness of the outer membrane of $A$. muciniphila, b: Diameter of $A$. muciniphila. The brain heart infusion broth (BHI), porcine mucin (PM), human mucin (HM), BHI+ porcine mucin (BPM), and BHI+ human mucin (BHM). The data are shown as the mean \pm SD $(n=4)$ and analyzed using one-way ANOVA with Tukey's test, ${ }^{*} p<0.05,{ }^{* *} p<0.01$

(acetic acid and butyric acid) on PM and HM. Studies showed that BCFAs like isobutyrate acid and isovalerate acid, were derived from the fermentation of branched-chain amino acids. In contrast to straight chain SCFAs, these compounds were considered detrimental to colonic and metabolic health [52, 53]. Compared with SCFAs, these compounds were considered harmful to the colon and metabolic health. Isobutyric acid and isovaleric acid were produced on $\mathrm{BHI}, \mathrm{BPM}$ and BHM. The contents of isobutyrate acid and isovalerate acid on BPM and BHM were relatively high, indicating that the glucose or mucin used by $A$. muciniphila was used simultaneously with glucose.

Outer membrane protein of A. muciniphila had a specific protein named Amuc_1100, which can maintain a stable state at the temperature of pasteurization and improve the intestinal barrier [14]. Through daily quantitative A. muciniphila feeding to mice, A. muciniphila can offset the increase in body weight and fat caused by high-fat diet (HFD) and improve glucose tolerance and insulin resistance, indicating that Amuc_1100 from pasteurization A. muciniphila outer membrane protein had beneficial effects on HFD- induced metabolic syndrome. Using five different media, we quantitatively detected the content of the outer membrane protein of $A$. muciniphila. Results showed the concentration of the outer membrane protein significantly increased in the batches grown on media containing only PM or HM. For these batches, the Amuc_1100 protein of A. muciniphila increased accordingly (Supplementary Fig. 1). The result provided an alternative way for the improvement of Amuc_1100 protein content of A. muciniphila outer membrane.

The cell morphology and cell size were observed by electron microscope. Found that the cell size differed depending on the medium under dynamic culture and static culture. Derrien et al. found that using porcine mucin medium under static culture [6], A. muciniphila could grow in single cells or in pairs and rarely grow in chains; it usually forms aggregates, and a translucent layer of material could be observed. Using BHI, this phenomenon was rarely observed, and cells appeared alone or in pairs but rarely in groups [31]. The results were different from our discoveries because of the culture methods (the dynamic culture was carried out in this study). 


\section{Conclusions}

Dietary changes can reveal the products and functions of the gut microbiota and their influence on human health. This study explained the growth, metabolism, and morphology of Akkermansia muciniphila in different nutrient media with an in vitro advanced bionic intestinal reactor. Our results indicated that the nutritional composition has a presumptive relationship with $A$. muciniphila biomass, outer membrane protein concentration and thickness, and cell diameter. At conditions containing mucin as sole carbon and nitrogen sources, the metabolites of $A$. muciniphila were acetic and butyric acids. This study provided a certain reference for the mechanism of $A$. muciniphila action in the host.

\section{Methods}

\section{Strains and media}

A. muciniphila AT56 were isolated from Chinese human feces. 16S rDNA sequencing showed that A. muciniphilas AT56 had 99\% similarity with the type stain A. muciniphila ATCC BAA-835. Phylogenetic information for the type strain was reported in NCBI Sequence Read Archive (SRA) database with the Bioproject number PRJNA331216.

The BHI contained $10.0 \mathrm{~g} / \mathrm{L}$ tryptone, $2.5 \mathrm{~g} / \mathrm{L}$ dibasic sodium phosphate, $17.5 \mathrm{~g} / \mathrm{L}$ beef heart infusion, $5.0 \mathrm{~g} / \mathrm{L}$ sodium chloride, and $2.0 \mathrm{~g} / \mathrm{L}$ glucose and was maintained at $\mathrm{pH} 6.5$.

The PM contained $4 \mathrm{~g} / \mathrm{L}$ porcine mucin, $2.5 \mathrm{~g} / \mathrm{L}$ disodium hydrogen phosphate, and $5.0 \mathrm{~g} / \mathrm{L}$ sodium chloride and was maintained at pH 6.5 (porcine mucin, Kuer, Beijing).

The HM contained $4 \mathrm{~g} / \mathrm{L}$ human mucin, $2.5 \mathrm{~g} / \mathrm{L}$ disodium hydrogen phosphate, and $5.0 \mathrm{~g} / \mathrm{L}$ sodium chloride and was maintained at pH 6.5 (human mucin, extracted from pancreatic myxoma).

The BPM contained $10.0 \mathrm{~g} / \mathrm{L}$ tryptone, $2.5 \mathrm{~g} / \mathrm{L}$ disodium hydrogen phosphate, $17.5 \mathrm{~g} / \mathrm{L}$ bovine heart dip powder, $5.0 \mathrm{~g} / \mathrm{L}$ sodium chloride, $2.0 \mathrm{~g} / \mathrm{L}$ glucose, and 4 $\mathrm{g} / \mathrm{L}$ pig-derived mucin and was maintained at $\mathrm{pH} 6.5$.

The BHM contained $10.0 \mathrm{~g} / \mathrm{L}$ tryptone, $2.5 \mathrm{~g} / \mathrm{L}$ disodium hydrogen phosphate, $17.5 \mathrm{~g} / \mathrm{L}$ bovine heart dip powder, $5.0 \mathrm{~g} / \mathrm{L}$ sodium chloride, $2.0 \mathrm{~g} / \mathrm{L}$ glucose, and 4 $\mathrm{g} / \mathrm{L}$ human mucin and was maintained at $\mathrm{pH} 6.5$.

All media had the same concentration, magnetically stirred for $2 \mathrm{~h}$, mixed well, and sterilized at $121^{\circ} \mathrm{C}$ for $30 \mathrm{~min}$ before use (Mucins was filtered by a $0.22 \mu \mathrm{m}$ water membrane to isolate bacteria).

Our study was performed in accordance with the principles of the Declaration of Helsinki with regard to ethical research involving human subjects, and the protocols were approved by Medical Ethics Committee of Affiliated Hospital of Jiangnan University (approval No. S2019-012-05).

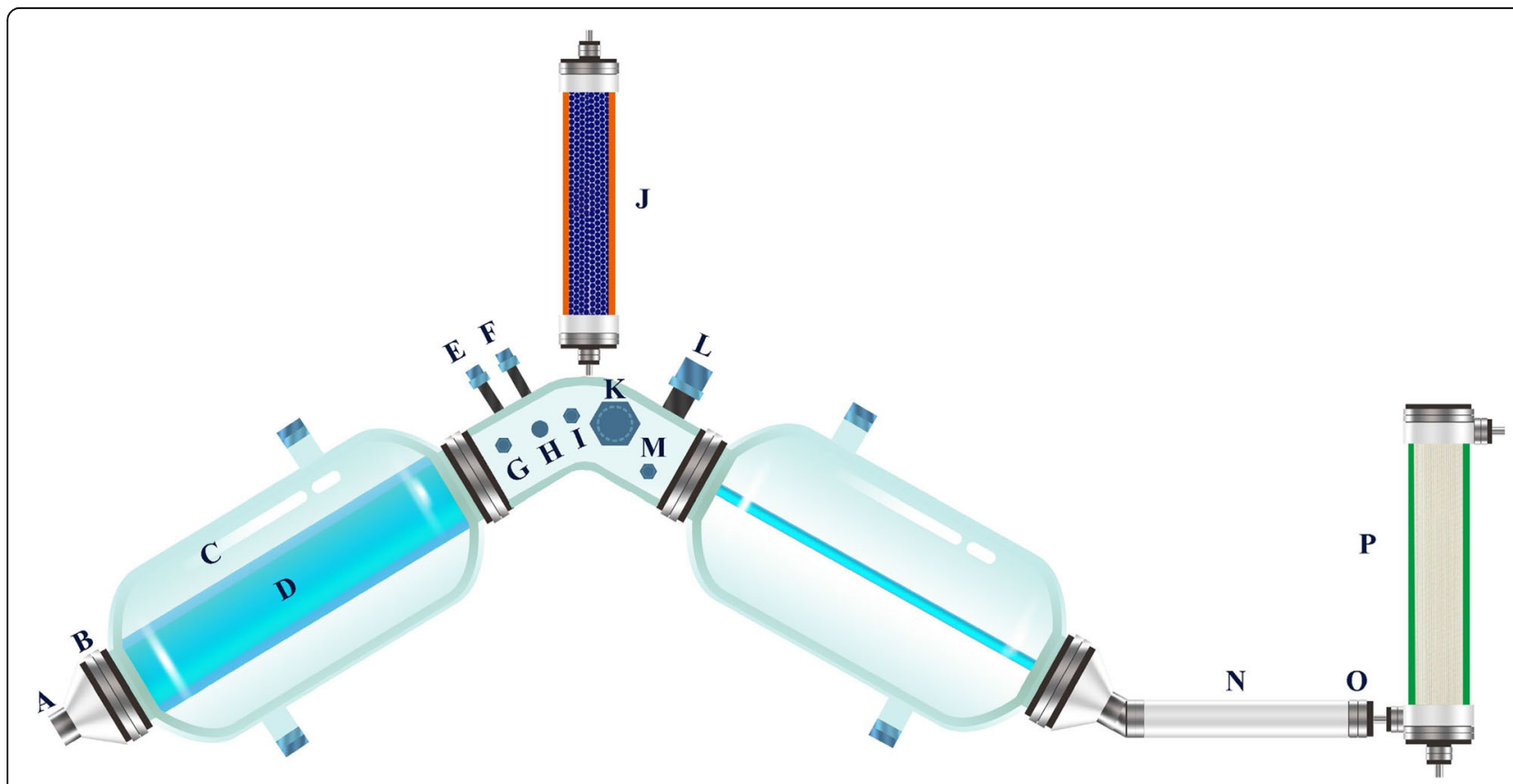

Fig. 6 Advanced bionic intestine reactor (ABIR): (A) discharge port, (B) sealing ring, (C) reaction bottle, (D) simulated large intestine, (E) alkaliadding port, $(F)$ feeding port, $(G)$ vacuum port, $(H)$ filling mixed gas port, (I) sample port, $(J)$ water suction device, $(K)$ sampling port, $(L)$ pH electrode, $(\mathrm{M}) \mathrm{OD}_{600}$ detection module, $(\mathrm{N})$ peristaltic tube, $(\mathrm{O})$ filter screen, $(\mathrm{P})$ dialysis device. (The figure is an original one, because the $\mathrm{ABIR}$ was designed by our team) 


\section{Device introduction}

An in vitro ABIR was developed to simulate the environment of A. muciniphila in the real large intestine [54]. As shown in Fig. 6, the bioreactor was composed of three interconnected reaction bottles with a flexible and transparent silica gel intestine that simulated the internal intestinal wall and the shape of the bionic intestine. The silica gel membrane between the reaction flask and the large intestine (Fig. 6d) was filled with deionized water at $37^{\circ} \mathrm{C}$. The simulated large intestine contracted and caused peristaltic waves by on line controlling the pressure of the water flow through a circulating water pump. Thus, the materials in the simulated large intestine were mixed and moved in the system. This mixing was better than the mixing done in the fermenter, where the phase separation of fluid and solid occurred. The reaction bottle was equipped with vacuum (Fig. $6 \mathrm{~g}$ ) and mixed gas (Fig. 6h) control devices to continuously maintain the oxygen-free environment in the simulation chamber. The system was also added with a water absorption device that simulated the function of the large intestine (Fig. 6j). The mixture in the cavity was absorbed to simulate the formation of feces. By continuously measuring the $\mathrm{pH}$ (Fig. 6l) and secreting alkaline solution (Fig. $6 \mathrm{e})$ to neutralize the acid, the system $\mathrm{pH}$ was maintained to prevent the accumulation of microbial metabolites. The system was equipped with a dialysis device, which consisted of hollow fiber nanofiltration membranes that filtered the metabolic products of microorganisms. Thus, the microbial metabolites in the reactor cavity were maintained at physiological concentrations.

\section{Dynamic and static culture methods}

The specific operation under dynamic culture was as follows. At first, the air tightness of the system was checked, and the whole reactor was immersed in water. The mixed gas aerator was opened to fill the reactor cavity with gas. If no bubble emerged, the air tightness of the system was good. The connection seals of each reaction bottle were detected to ensure that no bubble emerged. An aliquot of frozen stock culture of $\mathrm{A}$. muciniphila $(100 \mu \mathrm{L})$ was inoculated in $5 \mathrm{~mL}$ medium and incubated at $37^{\circ} \mathrm{C}$ for $24 \mathrm{~h}$ under strict anaerobic conditions (hydrogen, 5\%; carbon dioxide, 10\%; nitrogen, $85 \%)$. The configured medium was added into the ABIR through the sample port, placed in a sterilization pot maintained at $121^{\circ} \mathrm{C}$ for $30 \mathrm{~min}$, and cooled to $37^{\circ} \mathrm{C}$. The extraction valve was opened, and the air in the reactor was extracted. The extraction valve was closed, and the inflation valve was opened. Anaerobic gas (hydrogen, 5\%; carbon dioxide, 10\%; nitrogen $85 \%$ ) was poured into the reactor, and the process was repeated thrice for the reactor to have an anaerobic environment. The seed fluid flow of the incubated strain was added to the reactor cavity through a peristaltic pump. The reactor peristaltic system and the dynamic cultivation of the strain were started. In addition, the $\mathrm{OD}_{600}$ module (Fig. $6 \mathrm{~m}$ ) can be added to this reactor to monitor the $\mathrm{OD}_{600}$ value of the fermentation broth in real time. For static culture, the same cultured seed liquid $(5 \mathrm{~mL})$ was transferred to an anaerobic bottle with BPM medium volume of $300 \mathrm{~mL}$. The culture was carried out by static incubation for $48 \mathrm{~h}$ under the same conditions.

\section{Biomass determination}

The fermentation broth $(10 \mathrm{~mL})$ was collected and centrifuged, washed twice with deionized water, moved to the centrifuge tube after weighed, and centrifuged to obtain the cells. The cells were dried at $105^{\circ} \mathrm{C}$ and weighed [55].

\section{Determination of the short-chain fatty acids of metabolites}

The fermentation broth $(1 \mathrm{~mL})$ was collected, added with $10 \mu \mathrm{L} 2$-methylbutyric acid $(1 \mathrm{M})$ as an internal standard, and slowly added with $250 \mu \mathrm{L}$ concentrated hydrochloric acid. The mixture was mixed well, added with $1 \mathrm{~mL}$ diethyl ether, vortexed for $1 \mathrm{~min}$, and allowed to stand until the organic and the water phases separated. The supernatant (organic phase) was carefully collected, added with anhydrous sodium sulfate, vortexed, and passed through a $0.22 \mu \mathrm{m}$ organic filter. The supernatant $(5 \mu \mathrm{L})$ was injected into the Agilent 7890 gas chromatograph equipped with an electron capture detector (Agilent, USA), which was used to determine the short-chain fatty acids of $A$. muciniphila.

The gas chromatographic conditions were as follows: instrument, Agilent-7890A; chromatography column, HP-INNOWAX; detector temperature, $250^{\circ} \mathrm{C}$; inlet temperature, $220^{\circ} \mathrm{C}$; flow rate, $1.5 \mathrm{~mL} / \mathrm{min}$; split ratio, 1 : 20 ; heating program, $60^{\circ} \mathrm{C}-190^{\circ} \mathrm{C}, 4 \mathrm{~min}$; and injection volume, $5 \mu \mathrm{L}$.

\section{Bacterial outer membrane protein extraction method and concentration determination}

A. muciniphila was subjected to outer membrane protein extraction using the bacterial outer membrane protein extraction kit (BIOLABO HR0095, Beijing), and the protein content was estimated using the enhanced BCA protein assay kit (Beyotime Biotechnology, Beijing).

\section{FSEM and FTEM}

The cultured strains were washed with PBS buffer; fixed with $2.5 \%$ glutaraldehyde solution at $4{ }^{\circ} \mathrm{C}$ for $2 \mathrm{~h}$; washed again with PBS buffer; dehydrated using 30, 50, 70, 80, 90, and 100\% alcohol; dried; sprayed with gold; and lyophilized using the SU8200 (Japan) equipment. FSEM analysis was performed, and the morphology of $A$. 
muciniphila was observed at $3.0 \mathrm{KV} \times 10.0 \mathrm{k}$. The cell suspension was dropped on the copper grid and then dried at room temperature. TEM observations were taken by a transmission electronic microscope (JEMI010, Hitachi, Tokyo, Japan) in $120 \mathrm{kV}$.

\section{A. muciniphila outer membrane relative thickness and diameter measurement}

The TEM and SEM images were imported into the Adobe Photoshop CC 20.0.4, and the ruler tool was used to measure the relative thickness and diameter of the adventitia. Each cell was measured four times at different locations and the averaged values were presented.

\section{Statistical analysis}

Data were expressed as mean \pm SEM. Differences between the two groups were assessed using the unpaired twotailed Student $t$ test. The data sets that involved more than two groups were assessed using ANOVA. In the figures, data with * were significantly different at $p<0.05$ in accordance with posthoc ANOVA. Data were analyzed using the GraphPad Prism version 8.00 for Windows (GraphPad Software).

Statistical comparisons were indicated with *,**, and **** for $p<0.05, p<0.01$, and $p<0.001$, respectively.

\section{Supplementary Information}

The online version contains supplementary material available at https:/doi. org/10.1186/s12866-021-02111-7.

Additional file 1: Figure S1. Amuc_1100 Western blot. In this figure, the brain heart infusion broth $(\mathrm{BHI})$, porcine mucin $(\mathrm{PM})$, human mucin (HM).

\section{Abbreviations}

ABIR: Advanced bionic intestinal reactor; PM: Porcine mucin media; HM: Human mucin media; BHI: Brain heart infusion broth; BPM: BHI + porcine mucin; BHM: $\mathrm{BH}$ + human mucin; SCFAs: Short-chain fatty acids; BCFAs: Branched-chain fatty acids; SEM: Scanning electron microscope; TEM: Transmission Electron Microscope

\section{Acknowledgements}

Not applicable.

\section{Authors' contributions}

Conceived and designed research: MJG, ZTL and XBZ. Performed the experiments: ZTL, GAH, LZ and YJ. Analyzed the data: MJG, ZTL and XBZ. Contributed reagents/materials/analytical tools: ZTL, ZLS, MJG and XBZ. All authors read and approved the final manuscript.

\section{Funding}

This research was supported by National Key R\&D Program of China (2017YF0400302), and the National first-class discipline program of light industry technology and engineering (LITE2018-17), and Jiangsu Province Modern Agriculture Key Project (BE2018367) and the Priority Academic Program Development of Jiangsu Higher Education Institutions, the 111 Project (No. 111-2-06) is gratefully acknowledged. The first two funds provide the reagent and test fees contributing to the design of this study, data analysis and the writing of this manuscript. The rest of funds provide the fees of collection materials, language polishing, etc., contributing to the data analysis, the writing and the publication of this manuscript.

\section{Availability of data and materials}

165 rDNA sequencing data for Akkermansia muciniphila were deposited into NCBI Sequence Read Archive (SRA) database with the Bioproject number PRJNA331216 (https://www.ncbi.nlm.nih.gov/bioproject/331216).

\section{Ethics approval and consent to participate}

The experimental protocol was established, according to the ethical guidelines of the Helsinki Declaration and was approved by the Human Ethics Committee of Affiliated Hospital of Jiangnan University (approval No. S2019-012-05). Written informed consent of the subject was obtained.

\section{Consent for publication}

Not applicable.

\section{Competing interests}

The authors declare that they have no competing interests.

\section{Author details}

${ }^{1}$ Key Laboratory of Carbohydrate Chemistry and Biotechnology, Ministry of Education, School of Biotechnology, Jiangnan University, Wuxi 214122, China. ${ }^{2}$ Wuxi Galaxy Biotech Co. Ltd., Wuxi 214125, China. ${ }^{3}$ Suzhou Institute of Biomedical Engineering and Technology, Chinese Academy of Sciences, Suzhou 215163, China.

Received: 10 October 2020 Accepted: 7 February 2021

Published online: 23 February 2021

\section{References}

1. Cani PD, de Vos WM. Next-generation beneficial microbes: the case of Akkermansia muciniphila. Front Microbiol. 2017;8:1765.

2. Zhai Q, Feng S, Arjan N, Chen W. A next generation probiotic, Akkermansia muciniphila. Crit Rev Food Sci Nutr. 2019;59(19):3227-36.

3. Zhang T, Li Q, Cheng L, Buch H, Zhang F. Akkermansia muciniphila is a promising probiotic. Microb Biotechnol. 2019;12(6):1109-25.

4. van der Ark KCH, Aalvink S, Suarez-Diez M, Schaap PJ, de Vos WM, Belzer C. Model-driven design of a minimal medium for Akkermansia muciniphila confirms mucus adaptation. Microb Biotechnol. 2018;11(3):476-85.

5. Zhou K. Strategies to promote abundance of Akkermansia muciniphila, an emerging probiotics in the gut, evidence from dietary intervention studies. J Funct Foods. 2017:33:194-201.

6. Derrien M, Vaughan EE, Plugge CM, de Vos WM. Akkermansia muciniphila gen. Nov., sp. nov., a human intestinal mucin-degrading bacterium. Int. J. Syst. Evol. Microbiol. 2004;54(Pt 5):1469-76.

7. Le Poul E, Loison C, Struyf S, Springael JY, Lannoy V, Decobecq ME, Brezillon S, Dupriez V, Vassart G, Van Damme J, Parmentier M, Detheux M. Functional characterization of human receptors for short chain fatty acids and their role in polymorphonuclear cell activation. J Biol Chem. 2003;278(28):25481-9.

8. Koecher KJ, Noack JA, Timm DA, Klosterbuer AS, Thomas W, Slavin JL. Estimation and interpretation of fermentation in the gut: coupling results from a $24 \mathrm{~h}$ batch in vitro system with fecal measurements from a human intervention feeding study using fructo-oligosaccharides, inulin, gum acacia, and pea fiber. J Agric Food Chem. 2014;62(6):1332-7.

9. Jonathan MC, van den Borne JJGC, van Wiechen P, Souza da Silva C, Schols $\mathrm{HA}$, Gruppen $\mathrm{H}$. in vitro fermentation of 12 dietary fibres by faecal inoculum from pigs and humans. Food Chem. 2012;133(3):889-97.

10. Holck J, Hjernø K, Lorentzen A, Vigsnæs LK, Hemmingsen L, Licht TR, Mikkelsen JD, Meyer AS. Tailored enzymatic production of oligosaccharides from sugar beet pectin and evidence of differential effects of a single DP chain length difference on human faecal microbiota composition after in vitro fermentation. Process Biochem. 2011;46(5):1039-49.

11. Chassaing B, Koren O, Goodrich JK, Poole AC, Srinivasan S, Ley RE, Gewirtz AT. Dietary emulsifiers impact the mouse gut microbiota promoting colitis and metabolic syndrome. Nature. 2015;519(7541):92-6.

12. Dao MC, Everard A, Aron-Wisnewsky J, Sokolovska N, Prifti E, Verger EO, Kayser BD, Levenez F, Chilloux J, Hoyles L, Consortium MI-O, Dumas ME, Rizkalla SW, Dore J, Cani PD, Clement K. Akkermansia muciniphila and improved metabolic health during a dietary intervention in obesity: relationship with gut microbiome richness and ecology. Gut. 2016;65(3):426-36.

13. Everard A, Belzer C, Geurts L, Ouwerkerk JP, Druart C, Bindels LB, Guiot $Y$, Derrien M, Muccioli GG, Delzenne NM, de Vos WM, Cani PD. Cross-talk 
between Akkermansia muciniphila and intestinal epithelium controls dietinduced obesity. Proc Natl Acad Sci U S A. 2013;110(22):9066-71.

14. Plovier H, Everard A, Druart C, Depommier C, Van Hul M, Geurts L, Chilloux J, Ottman N, Duparc T, Lichtenstein L, Myridakis A, Delzenne NM, Klievink J, Bhattacharjee A, van der Ark KC, Aalvink S, Martinez LO, Dumas ME, Maiter D, Loumaye A, Hermans MP, Thissen JP, Belzer C, de Vos WM, Cani PD. A purified membrane protein from Akkermansia muciniphila or the pasteurized bacterium improves metabolism in obese and diabetic mice. Nat. Med. 2017;23(1):107-13.

15. Qin J, Li Y, Cai Z, Li S, Zhu J, Zhang F, Liang S, Zhang W, Guan Y, Shen D, Peng $Y$, Zhang D, Jie Z, Wu W, Qin Y, Xue W, Li J, Han L, Lu D, Wu P, Dai Y, Sun X, Li Z, Tang A, Zhong S, Li X, Chen W, Xu R, Wang M, Feng Q, Gong M, Yu J, Zhang Y, Zhang M, Hansen T, Sanchez G, Raes J, Falony G, Okuda S, Almeida M, LeChatelier E, Renault P, Pons N, Batto JM, Zhang Z, Chen H, Yang R, Zheng W, Li S, Yang H, Wang J, Ehrlich SD, Nielsen R, Pedersen O, Kristiansen K, Wang J. A metagenome-wide association study of gut microbiota in type 2 diabetes. Nature. 2012;490(7418):55-60.

16. Zhao S, Liu W, Wang J, Shi J, Sun Y, Wang W, Ning G, Liu R, Hong J. Akkermansia muciniphila improves metabolic profiles by reducing inflammation in chow diet-fed mice. J Mol Endocrinol. 2017;58(1):1-14

17. Depommier C, Everard A, Druart C, Plovier H, Van Hul M, Vieira-Silva S, Falony G, Raes J, Maiter D, Delzenne NM, de Barsy M, Loumaye A, Hermans MP, Thissen JP, de Vos WM, Cani PD. Supplementation with Akkermansia muciniphila in overweight and obese human volunteers: a proof-of-concept exploratory study. Nat Med. 2019;25(7):1096-103.

18. Png CW, Linden SK, Gilshenan KS, Zoetendal EG, McSweeney CS, Sly LI, McGuckin MA, Florin TH. Mucolytic bacteria with increased prevalence in IBD mucosa augment in vitro utilization of mucin by other bacteria. Am J Gastroenterol. 2010;105(11):2420-8.

19. Zhai R, Xue X, Zhang L, Yang X, Zhao L, Zhang C. Strain-specific antiinflammatory properties of two Akkermansia muciniphila strains on chronic colitis in mice. Front Cell Infect Microbiol. 2019:9:239.

20. Wang L, Christophersen CT, Sorich MJ, Gerber JP, Angley MT, Conlon MA. Low relative abundances of the mucolytic bacterium Akkermansia muciniphila and Bifidobacterium spp. in feces of children with autism. Appl Environ Microbiol. 2011;77(18):6718-21.

21. Candela M, Rampelli S, Turroni S, Severgnini M, Consolandi C, Bellis GD, Masetti R, Ricci G, Pession A, Brigidi P. Unbalance of intestinal microbiota in atopic children. BMC Microbiol. 2012;12(95):2-9.

22. Blacher E, Bashiardes S, Shapiro H, Rothschild D, Mor U, Dori-Bachash M, Kleimeyer C, Moresi C, Harnik Y, Zur M, Zabari M, Brik RB, Kviatcovsky D, Zmora N, Cohen Y, Bar N, Levi I, Amar N, Mehlman T, Brandis A, Biton I, Kuperman Y, Tsoory M, Alfahel L, Harmelin A, Schwartz M, Israelson A, Arike $L$, Johansson MEV, Hansson GC, Gotkine M, Segal E, Elinav E. Potential roles of gut microbiome and metabolites in modulating ALS in mice. Nature. 2019;572(7770):474-80.

23. Barcena C, Valdes-Mas R, Mayoral P, Garabaya C, Durand S, Rodriguez F, Fernandez-Garcia MT, Salazar N, Nogacka AM, Garatachea N, Bossut N, Aprahamian F, Lucia A, Kroemer G, Freije JMP, Quiros PM, Lopez-Otin C. Healthspan and lifespan extension by fecal microbiota transplantation into progeroid mice. Nat Med. 2019;25(8):1234-42.

24. Olson CA, Vuong HE, Yano JM, Liang QY, Nusbaum DJ, Hsiao EY. The gut microbiota mediates the anti-seizure effects of the Ketogenic diet. Cell. 2018;173(7): 1728-41 e13.

25. Li J, Zhao F, Wang Y, Chen J, Tao J, Tian G, Wu S, Liu W, Cui Q, Geng B, Zhang W, Weldon R, Auguste K, Yang L, Liu X, Chen L, Yang X, Zhu B, Cai J. Gut microbiota dysbiosis contributes to the development of hypertension. Microbiome. 2017:5(1):14

26. Routy $B$, Chatelier EL, Derosa L, Duong CPM, Alou MT, Daillère R, Fluckiger A, Messaoudene M, Rauber C, Roberti MP, Fidelle M. Gut microbiome influences efficacy of PD-1-based immunotherapy against epithelial tumors. Science. 2018;259:91-7.

27. ljssennagger N, Belzer C, Hooiveld GJ, Dekker J, van Mil SW, Muller M, Kleerebezem M, van der Meer R. Gut microbiota facilitates dietary hemeinduced epithelial hyperproliferation by opening the mucus barrier in colon. Proc Natl Acad Sci U S A. 2015;112(32):10038-43.

28. Sanna S, van Zuydam NR, Mahajan A, Kurilshikov A, Vich Vila A, Võsa U, Mujagic Z, Masclee AAM, Jonkers DMAE, Oosting M, Joosten LAB, Netea MG, Franke L, Zhernakova A, Fu J, Wijmenga C, McCarthy MI. Causal relationships among the gut microbiome, short-chain fatty acids and metabolic diseases. Nat Genet. 2019;51(4):600-5.
29. Guo X, Zhang J, Wu F, Zhang M, Yi M, Peng Y. Different subtype strains of Akkermansia muciniphila abundantly colonize in southern China. J Appl Microbiol. 2016;120(2):452-9.

30. Marcial-Coba MS, Saaby L, Knochel S, Nielsen DS. Dark chocolate as a stable carrier of microencapsulated Akkermansia muciniphila and Lactobacillus casei. FEMS Microbiol Lett. 2019;366:fny290. https://doi.org/10.1093/femsle/ fny290.

31. Derrien M, Collado MC, Ben-Amor K, Salminen S, de Vos WM. The Mucin degrader Akkermansia muciniphila is an abundant resident of the human intestinal tract. Appl Environ Microbiol. 2008;74(5):1646-8.

32. Bharti SK, Krishnan S, Kumar A, Gupta AK, Ghosh AK, Kumar A. Mechanismbased antidiabetic activity of Fructo- and isomalto-oligosaccharides: validation by in vivo, in silico and in vitro interaction potential. Process Biochem. 2015;50(2):317-27.

33. Ramasamy US, Venema K, Schols HA, Gruppen H. Effect of soluble and insoluble fibers within the in vitro fermentation of chicory root pulp by human gut bacteria. J Agric Food Chem. 2014;62(28):6794-802.

34. Chia LW, Hornung BVH, Aalvink S, Schaap PJ, de Vos WM, Knol J, Belzer C. Deciphering the trophic interaction between Akkermansia muciniphila and the butyrogenic gut commensal Anaerostipes caccae using a metatranscriptomic approach. Antonie Van Leeuwenhoek. 2018;111(6):859-73.

35. Belzer C, Chia LW, Aalvink S, Chamlagain B, Piironen V, Knol J, de Vos WM. Microbial Metabolic Networks at the Mucus Layer Lead to Diet-Independent Butyrate and Vitamin B12 Production by Intestinal Symbionts. mBio. 2017; 8(5):e00770-17.

36. Ottman N, Davids M, Suarez-Diez M, Boeren S, Schaap PJ, Martins Dos Santos VAP, Smidt H, Belzer C, de Vos WM. Genome-Scale Model and Omics Analysis of Metabolic Capacities of Akkermansia muciniphila Reveal a Preferential Mucin-Degrading Lifestyle. Appl Environ Microbiol. 2017;83(18): e01014-7.

37. Wang M, Zhang XY, Guo RR, Cai ZP, Hu XC, Chen H, Wei S, Voglmeir J, Liu L. Cloning, purification and biochemical characterization of two beta- $\mathrm{N}$ acetylhexosaminidases from the mucin-degrading gut bacterium Akkermansia muciniphila. Carbohydr Res. 2018;457:1-7.

38. Collado MC, Derrien M, Isolauri E, de Vos WM, Salminen S. Intestinal integrity and Akkermansia muciniphila, a mucin-degrading member of the intestinal microbiota present in infants, adults, and the elderly. Appl Environ Microbiol. 2007;73(23):7767-70.

39. Ouwerkerk JP, van der Ark KCH, Davids M, Claassens NJ, Finestra TR, de Vos WM, Belzer C. Adaptation of Akkermansia muciniphila to the Oxic-anoxic Interface of the mucus layer. Appl Environ Microbiol. 2016;82(23):6983-93.

40. Reid DT, Eller LK, Nettleton JE, Reimer RA. Postnatal prebiotic fibre intake mitigates some detrimental metabolic outcomes of early overnutrition in rats. Eur J Nutr. 2016;55(8):2399-409.

41. Everard A, Lazarevic V, Derrien M, Girard M, Muccioli GG, Neyrinck AM, Possemiers S, Holle AV. Responses of Gut Microbiota and Glucose and Lipid Metabolism to Prebiotics in Genetic Obese and Diet-Induced LeptinResistant Mice. Diabetes. 2011;60:2775-86.

42. Zhou ZY, Ren LW, Zhan P, Yang HY, Chai DD, Yu ZW. Metformin exerts glucose-lowering action in high-fat fed mice via attenuating endotoxemia and enhancing insulin signaling. Acta Pharmacol Sin. 2016;37(8):1063-75.

43. Lee $H$, Ko G. Effect of metformin on metabolic improvement and gut microbiota. Appl Environ Microbiol. 2014;80(19):5935-43.

44. Anhe FF, Nachbar RT, Varin TV, Vilela V, Dudonne S, Pilon G, Fournier M, Lecours MA, Desjardins Y, Roy D, Levy E, Marette A. A polyphenol-rich cranberry extract reverses insulin resistance and hepatic steatosis independently of body weight loss. Mol Metab. 2017;6(12):1563-73.

45. Anhe FF, Roy D, Pilon G, Dudonne S, Matamoros S, Varin TV, Garofalo C, Moine Q, Desjardins Y, Levy E, Marette A. A polyphenol-rich cranberry extract protects from diet-induced obesity, insulin resistance and intestinal inflammation in association with increased Akkermansia spp. population in the gut microbiota of mice. Gut. 2015;64(6):872-83.

46. Roopchand DE, Carmody RN, Kuhn P, Moskal K, Rojas-Silva P, Turnbaugh PJ, Raskin I. Dietary Polyphenols Promote Growth of the Gut Bacterium Akkermansia muciniphila and Attenuate High-Fat Diet-Induced Metabolic Syndrome. Diabetes. 2015:64:2847-58.

47. Anhe FF, Pilon G, Roy D, Desjardins Y, Levy E, Marette A. Triggering Akkermansia with dietary polyphenols: A new weapon to combat the metabolic syndrome? Gut Microbes. 2016;7(2):146-53.

48. Alard J, Lehrter V, Rhimi M, Mangin I, Peucelle V, Abraham AL, Mariadassou M, Maguin E, Waligora-Dupriet AJ, Pot B, Wolowczuk I, Grangette C. 
Beneficial metabolic effects of selected probiotics on diet-induced obesity and insulin resistance in mice are associated with improvement of dysbiotic gut microbiota. Environ Microbiol. 2016;18(5):1484-97.

49. Martinez-Gutierrez F, Ratering S, Juárez-Flores B, Godinez-Hernandez C Geissler-Plaum R, Prell F, Zorn H, Czermak P, Schnell S. Potential use of Agave salmiana as a prebiotic that stimulates the growth of probiotic bacteria. Lwt. 2017;84:151-9.

50. Ashrafian F, Shahriary A, Behrouzi A, Moradi HR, Keshavarz Azizi Raftar S, Lari A, Hadifar S, Yaghoubfar R, Ahmadi Badi S, Khatami S, Vaziri F, Siadat SD. Akkermansia muciniphila-Derived Extracellular Vesicles as a Mucosal Delivery Vector for Amelioration of Obesity in Mice. Front Microbiol. 2019;10:2155.

51. Caesar R, Tremaroli V, Kovatcheva-Datchary P, Cani PD, Backhed F. Crosstalk between gut microbiota and dietary lipids aggravates WAT inflammation through TLR signaling. Cell Metab. 2015;22(4):658-68.

52. Liebisch G, Ecker J, Roth S, Schweizer S, Ottl V, Schott HF, Yoon H, Haller D, Holler E, Burkhardt R, Matysik S. Quantification of Fecal Short Chain Fatty Acids by Liquid Chromatography Tandem Mass Spectrometry-Investigation of Pre-Analytic Stability. Biomolecules. 2019;9((4):121.

53. Zhou L, Xie M, Yang F, Liu J. Antioxidant activity of high purity blueberry anthocyanins and the effects on human intestinal microbiota. Lwt. 2020;117.

54. Li Z-t, Zhu L, Zhang W-I, Zhan X-b, Gao M-j. New dynamic digestion model reactor that mimics gastrointestinal function. Biochem Eng J. 2020;154.

55. Ruan S, Luo J, Li Y, Wang Y, Huang S, Lu F, Ma H. Ultrasound-assisted liquidstate fermentation of soybean meal with Bacillus subtilis: effects on peptides content, ACE inhibitory activity and biomass. Process Biochem. 2020;91:73-82

\section{Publisher's Note}

Springer Nature remains neutral with regard to jurisdictional claims in published maps and institutional affiliations.

Ready to submit your research? Choose BMC and benefit from:

- fast, convenient online submission

- thorough peer review by experienced researchers in your field

- rapid publication on acceptance

- support for research data, including large and complex data types

- gold Open Access which fosters wider collaboration and increased citations

- maximum visibility for your research: over $100 \mathrm{M}$ website views per year

At $\mathrm{BMC}$, research is always in progress.

Learn more biomedcentral.com/submissions 